palliative care should be integrated as a routine element of all undergraduate medical and nursing professional education 'However, the provision of training in palliative medicine is variable both nationally and internationally.

Methods Initially organised as a Taskforce of the EAPC Steering Group on Medical Education and Training, the International Medical Education in Palliative Care (IMEP) project sought to examine how well current training prepares doctors to care for patients with palliative needs. EORTC structured translations of validated assessment tool were produced in French, German, Spanish and Italian, and piloted in participating countries. In addition, MD students from Aachen University, and $\mathrm{PhD}$ students from the University of Liverpool have developed further translations/assessments in China, India, Brazil, South Africa and Armenia. As a development, the 'Global IMEP Initiative' seeks to:

- pilot an electronic version of the assessment tools;

- coalesce existing data; and

- engage wider international collaboration to establish baselines on the preparedness of newly qualified doctors to practice palliative care.

Results To date, electronic versions of tools have been produced in English and German, structured to be accessible across computer, tablet and smartphone formats. Piloting of the electronic tool is ongoing in the UK, Germany and Israel. Preliminary data indicates that the electronic format is user friendly, transferable across languages and increases return rate.

Conclusion The IMEP electronic assessment tools are amenable to international translation and dissemination. Data from established national and international baselines will assist in the further promotion of the integration of training in palliative medicine within existing curricula.

\section{NON-MALIGNANT ADVANCE CARE PLANNING - CREATING A COMMUNITY OF PRACTICE}

Louise Gilhooley, Derek Willis, Alice O'Connor. Severn Hospice, Shropshire Council

\subsection{6/bmjspcare-2018-ASPabstracts.58}

Introduction Advance Care Planning (ACP) in non-malignant disease is recognised as helping to improve care and outcomes for patients at end of life. These discussions can be challenging for both patients and healthcare professionals. Education in ACP locally in Shropshire was needed, but how to deliver this and share good practice was the object of this course.

Methods A two day conference was designed and delivered in May 2017 in Shropshire. Over 60 delegates attended both days. Day one was predominantly didactic lecture based in design covering the Law and Mental Capacity Act, relative's perspectives (a pre-recorded video) and specific case presentations. Day two used multi-professional small group discussions in five disease specific workshops including heart failure and renal failure. The results of discussions were used to inform future policy and practice.

Results Both days were oversubscribed and positively evaluated. Evaluations described an increase in confidence in approaching ACP discussions and the value of collaborative working and sharing of information. Overall the workshop delivery was particularly beneficial. For example, in the heart failure group it allowed recognition of areas of strength and development. It highlighted the need to identify projects that are going to: a) Increase the knowledge of both sets of Clinical Nurse Specialists (heart failure and palliative) b) Identify patients so that their location and what is happening is known to various teams

The overall aim to encourage hospice and heart failure professions to work collaboratively in symptomatic management of patients with heart failure.

Conclusion As a result of this conference streams of education in renal and heart failure are planned. Closer working relationships have been formed including a renal palliative MDT and heart failure MDT. The conference is to be repeated in 2018 using a similar program.

\section{INTEGRATED RENAL PALLIATIVE CARE - CREATING A COMMUNITY OF PRACTICE}

Louise Gilhooley, Sunita Nair, Elaine Gibson, Rebecca Verling. Severn Hospice, Shrewsbury and Telford NHS Trust

\subsection{6/bmjspcare-2018-ASPabstracts.59}

Introduction It is increasingly recognised that patients with end stage renal failure can have needs similar to those with advanced cancer. This is particularly relevant in patients who have opted for conservative management or who wish to withdraw from renal replacement therapy (RRT) and in those patients who are deteriorating despite RRT. In Shropshire a renal palliative multidisciplinary team (MDT) has been formed and it is recognised that mutual education is important in delivering a good service to these patients.

Methods Joint workshops were held to identify learning needs and a two day programme was then designed and delivered in June/July 2017. Day one covered aspects of mortality/morbidity of RRT in an ageing population, conservative care, psychological adaptations and symptom management. Day two delivered communication skills training specific to renal scenarios, using simulated patients.

Results The course was well attended and positively evaluated. Overall delegates reported an improved confidence in approaching end of life issues in renal patients and a recognition of the importance and benefit of a collaborative approach, with effective information sharing and ongoing coeducation. Recognised especially was the complexity of an ageing and increasing frail population of patients and a recognition of the psychological adaptations over time to renal failure. Evaluations for communication skills training revealed increased confidence in initiating and expanding conversations, especially around advance care planning and resuscitation.

Conclusion A greater understanding of the population we support and mutual education has resulted in closer working relationships within the renal palliative MDT. Dialysis end of life link nurses have planned spin-off educational sessions with frontline dialysis staff. The education programme is to be repeated in 2018 using a similar format. Similar streams of education in heart failure are planned. 